\title{
Hysteroscopic treatment of symptomatic adenomyoma
}

\author{
(D) Jin Yu1,2,3, (D) Duo Zhang1,2,3, (D) Wei Xia1,2,3, (D) Jian Zhang1,2,3 \\ 1Department of Obstetrics and Gynecology, International Peace Maternity and Child Health Hospital School of Medicine, \\ Shanghai Jiaotong University, Shanghai, China \\ 2Institute of Embryo-Fetal Original Adult Disease Affiliated to School of Medicine, Shanghai Jiaotong University, \\ Shanghai, China \\ ${ }^{3}$ Shanghai Key Laboratory of Embryo Original Diseases, Shanghai, China
}

\section{Abstract}

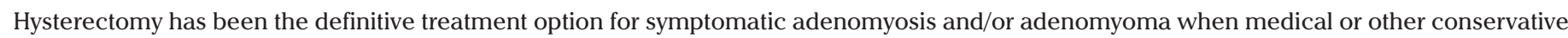

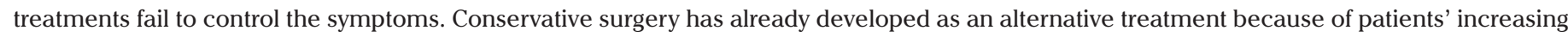

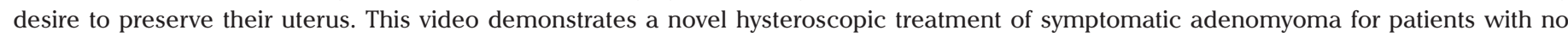
desire for fertility. (J Turk Ger Gynecol Assoc 2020; 21: 140-2)

Keywords: Adenomyoma, adenomyosis, hysteroscopy, hysteroscopic surgical procedures

Received: 13 April, 2019 Accepted: 20 June, 2019

\section{Introduction}

Adenomyosis is a kind of benign gynecologic disorder with the invasion of endometrial glands and stroma in the uterine myometrium, which results in pelvic pain, dysmenorrhea, and menorrhagia (1). The disease may be diffuse or focal with adenomyoma. Hysterectomy has been known as the primary treatment for adenomyosis and/or adenomyoma (2).

Traditionally, adenomyosis would be found incidentally in specimens obtained from uterine biopsies or hysterectomy and/or percutaneous ultrasound-based biopsies. Modern diagnostic imaging techniques, such as magnetic resonance imaging (MRI), which have high accuracy in identifying this kind of pathology, have led to conservative uterine-sparing treatments of adenomyosis and/or adenomyoma becoming efficacious and feasible $(3,4)$.

This video shows the hysteroscopic surgical procedures of two women with adenomyoma (Figure 1) requesting surgical management for the relief of symptoms and the preservation of the uterus, but with no desire for future fertility. These two patients both had heavy menstrual bleeding and severe dysmenorrhea. We used saline solution to dilate the uterine cavity and set the intrauterine pressure at $120 \mathrm{mmHg}$. The operation was performed with a transcervical resection resectoscope equipped with a $3 \mathrm{~mm}$ and $5 \mathrm{~mm}$ wide loop. The surgeon dilated the cervix to $9 \mathrm{~mm}$, then used a cutting loop to resect the lesions repeatedly and progressively. With color Doppler ultrasound guidance, the first step was to evaluate the features of the uterine cavity. Then, the surgeon used a cutting loop to progressively resect the lesions (Figure 2). The operation was completed with the appearance of the pink fasciculate structure of the myometrium. Tissue fragments were removed at intervals using ovum forceps. The specimens were sent for histologic analysis (Figure 3).

Follow-up was performed twice at 3-month intervals. The patient menstruated regularly. The postoperative visual analogue scale scores of menstrual blood volume and dysmenorrhea appeared to decline substantially. The uterine volume was evaluated using MRI 6 months later and was reduced by approximately $33 \%$. 

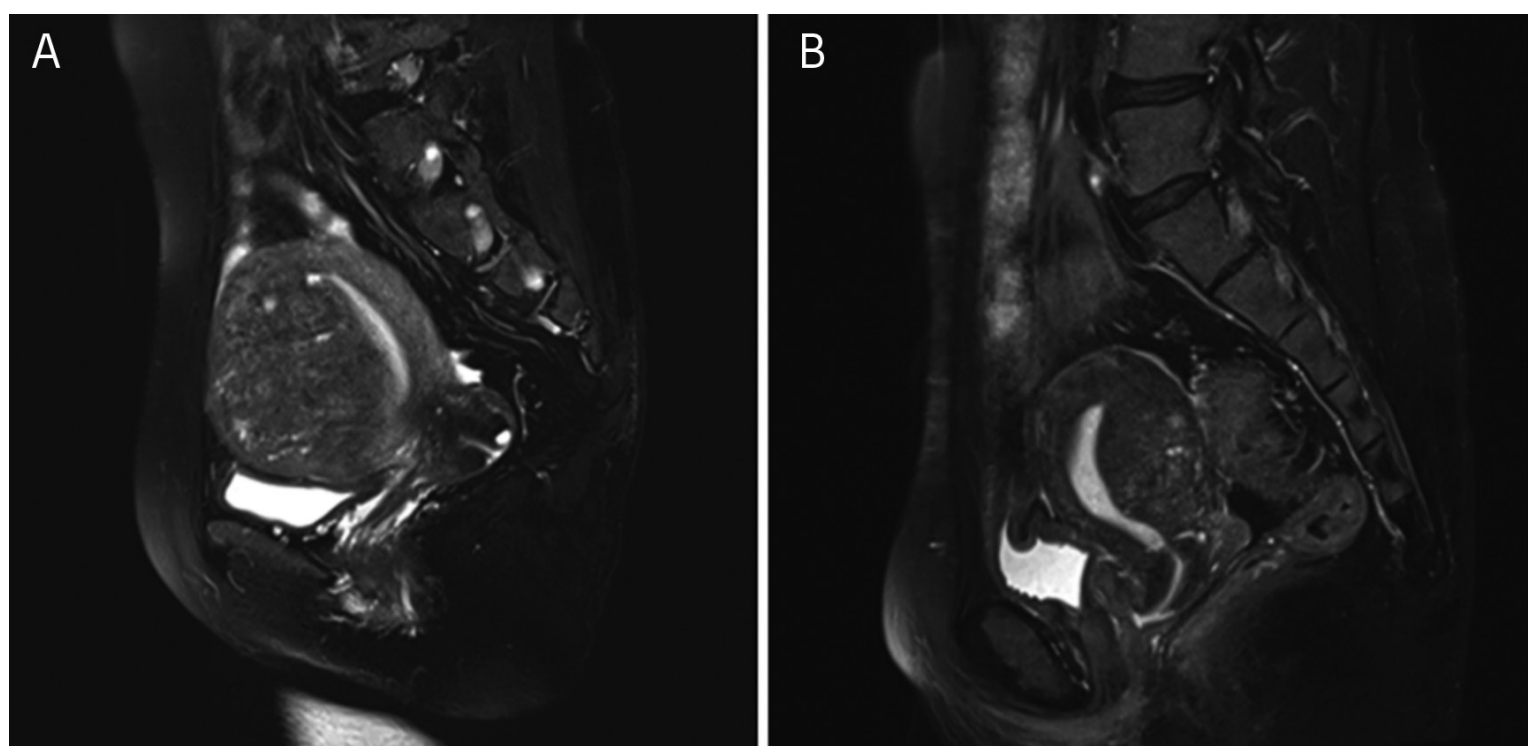

Figure 1. Magnetic resonance imaging imaging of adenomyosis

The adenomyotic lesions in case one were located in the anterior uterine wall (A), and the lesions in case two were located in the posterior wall (B)
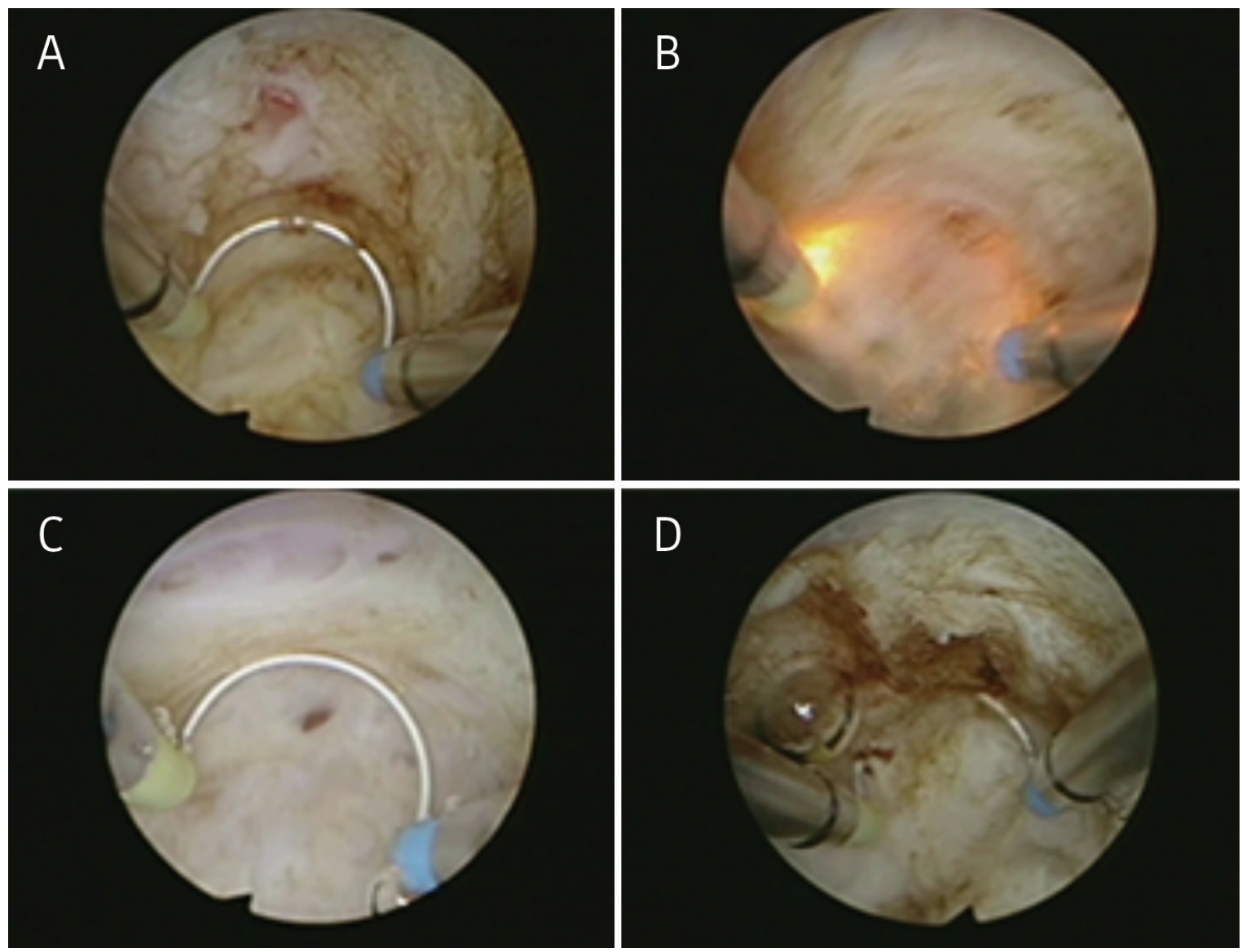

Figure 2. Surgical procedure

In case one, upon cutting the endometrium covering the adenomyotic lesions (A), pink ectopic endometrial lesions in the myometrium were exposed. (B) The ectopic endometrium and adenomyotic lesions were gradually excised from the myometrium. (C) During the resection of lesions, several intramural microcysts with a wide base were revealed. (D) Opening the microcyst resulted in the outflow composed mostly of old blood 


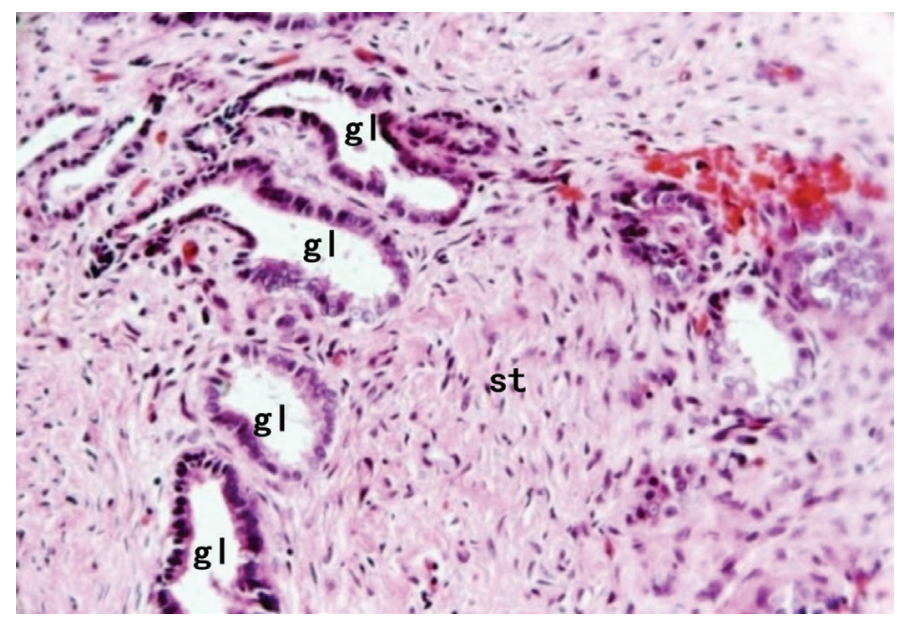

Figure 3. HE staining of adenomyosis St: Stromal cells, gl: Glands

Uterine perforation is the greatest risk associated with hysteroscopic resection surgery. For the duration of the procedure, the surgeon should pay particular attention to fluid management and prepare with solutions when fluid overload or hyponatremia is suspected. Hysteroscopic excision of uterine adenomyoma has the following benefits: The uterus is preserved and the symptoms of adenomyoma are improved; the minimally invasive operation takes a short time and patients recover quickly. Therefore, hysteroscopic excision can become an effective conservative treatment option for adenomyoma.

Video 1: https://www.doi.org/10.4274/jtgga.galenos.2019.2019.0062. video1

Conflict of Interest: No conflict of interest was declared by the authors.

Financial Disclosure: This work was supported by grants from the Shanghai Hospital Development Center (grant number SHDC12015121) and the Science and Technology Commission of Shanghai Municipality (grant numbers: 16411950502, 16411963200).

\section{References}

1. Xia W, Zhang D, Zhu Q, Zhang H, Yang S, Ma J, et al. Hysteroscopic excision of symptomatic myometrial adenomyosis: Feasibility and effectiveness. BJOG 2017; 124: 1615-20.

2. Tsui KH, Lee WL, Chen CY, Sheu BC, Yen MS, Chang TC, et al. Medical treatment for adenomyosis and/or adenomyoma. Taiwan J Obstet Gynecol 2014; 53: 459-65.

3. Grimbizis GF, Mikos T, Tarlatzis B. Uterus-sparing operative treatment for adenomyosis. Fertil Steril 2014; 101: 472-87.

4. Di Spiezio Sardo A, Calagna G, Santangelo F, Zizolfi B, Tanos $\mathrm{V}$, Perino A, et al. The role of hysteroscopy in the diagnosis and treatment of adenomyosis. Biomed Res Int 2017; 2017: 2518396. 